\title{
Four-Channel Label-Free Photonic Crystal Biosensor Using Nanocavity Resonators
}

\author{
Saeed OLYAEE $^{1^{*}}$, Samira NAJAFGHOLINEZHAD ${ }^{1}$, and \\ Hamed ALIPOUR BANAEI ${ }^{2}$
}

\author{
${ }^{1}$ Nano-photonics and Optoelectronics Research Laboratory (NORLab), Faculty of Electrical and Computer Engineering, \\ Shahid Rajaee Teacher Training University, Tehran, Iran \\ ${ }^{2}$ Electrical Department, Faculty of Engineering, Tabriz Branch, Islamic Azad University, Tabriz, Iran \\ ${ }^{*}$ Corresponding Author: Saeed OLYAEEＩ E-mail: s_olyaee@srttu.edu
}

\begin{abstract}
In this paper, we design and characterize a novel small size four-channel biosensor based on the two-dimensional photonic crystal with introducing waveguides and nano-cavities in the hexagonal lattice of air pores in the silicon slab. By removing a group of air pores, waveguides are achieved, and nano-cavities are shaped by modifying the radius of air pores. Highly parallel operation of this biosensor due to the special architecture is the capability of the designed structure. The biomaterials which are suspended in a liquid medium inside nano-cavities cause effective refractive index changes which lead to the resonant wavelength shift in the output terminal. According to results, with increasing the refractive index of nano-cavities, resonant wavelengths shifts to longer values. For biochemical sensing like DNA molecule and protein and for the refractive index detection, this novel designed biosensor can be utilized.
\end{abstract}

Keywords: Photonic crystal, four-channel biosensor, nano-cavity, waveguide

Citation: Saeed OLYAEE, Samira NAJAFGHOLINEZHAD, and Hamed ALIPOUR BANAEI, "Four-Channel Label-Free Photonic Crystal Biosensor Using Nanocavity Resonators," Photonic Sensors, DOI: 10.1007/s13320-013-0110-y.

\section{Introduction}

Photonic crystals are the periodic dielectric structures, containing the array of air pores in the silicon slab which have the light localizing capability. A forbidden frequency range that is called the photonic band gap (PBG) is one of the most important properties of these structures [1-4]. Photonic crystal sensors have applications in a wide measurement range of physical properties such as temperature, pressure, refractive index, bio-sensing [5-8]. Defects in the photonic crystal which break the periodicity of the structure are important for sensing applications because they help for localizing light and promoting the interaction of the light beam [9]. There are two basic schemes in optical sensing: the first one is fluorescent based sensing, and the second one is label-free sensing [10-12].

Label-free photonic crystal sensors are utilized for measuring the refractive index variation due to high manipulating of light propagation. For label-free detection, bio-molecules do not label with fluorescent tags, whereas the effective refractive index variation near the active sensing surface occurs in this way. This process is a way to measure the presence of the bio-molecules $[13,14]$. The

Received: 1 February 2013 / Revised version: 19 April 2013

C The Author(s) 2013. This article is published with open access at Springerlink.com 
presence of the biochemical material within selected holes especially defects, causes a spectral shift. Defects such as micro-cavities or nano-cavities based on photonic crystal structures reveal different sensitive and high quality factor sensors [15].

Two types of the sensing mechanism exist for the bio-sensing application. There are homogenous sensing and surface sensing schemes. In homogenous sensing, the effective refractive index (ERI) variation is due to the covered medium refractive index change. And in surface sensing, the binding mechanism changes the thickness of the sensing hole which leads to ERI modification [16].

S. H. Kwon et al. proposed a photonic crystal chemical sensor based on a cavity [17]. Also Wang et al. designed a refractive index sensor for biolayers and chemical sensing, consisting of a microcavity and two waveguides [18]. Hsiao and Lee investigated a photonic crystal nano-ring resonator as the biochemical sensor based on the single defect hole [15]. In order to overcome the single purpose operation of the sensor and amount of detected target molecules in a special time, a four-channel biosensor is regarded. On the contrary of other structures which can be utilized for sensing bio-molecules with one specific refractive index in a certain time, our novel designed biosensor can be used for sensing four specific refractive indices in a certain time; so a multichannel biosensor is constructed.

The photonic crystal biosensors are not interesting only for having the high sensitivity to local and bulk refractive indices $(\Delta \lambda / \Delta n)$. But rather, the small mode volume makes them more sensitive to the total mass amount which is important especially in bio-sensing applications. The small mode volume requires the small mass amount to reflect the resonant wavelength shift $(\Delta \lambda / N)$, where $N$ is the number of functionalized holes for sensing [19]. D. Q. Yang et al. proposed a photonic crystal sensor array with optimized 6 functionalized holes based on micro-cavities with a few mass sensitivity [19]. The advantages of nano-cavity to micro-cavity resonators are the highly localizing electromagnetic field and high quality factor and improvement in the light-matter interaction due to the small mode volume of the analyte.

In this paper, a four-channel biosensor based on the two-dimensional photonic crystal is proposed by introducing waveguides and nano-cavities. We present the structure analysis for bio-sensing applications based on the single sensing hole within the nano-cavity for each channel. With a system, the analyte is induced to nano-cavities in the two-dimensional photonic crystal. By flowing the analyte through selected holes, the refractive index of the nano-holes will change, and the transmission spectra of the structure vary which can be measured to determine the properties of the analyte. In this research, we designed an ultra small size novel multichannel biosensor with optimized geometry parameters based on nano-cavities.

\section{Designing multi-channel biosensor}

According to Fig. 1, the biosensor is constructed by modifying three parts, which is mentioned as follows. The first: by removing a row of air pores, the main waveguide which is called the bus waveguide [20] is created that is utilized for applying the Gaussian pulse and actuating resonant modes of any nano-cavities.

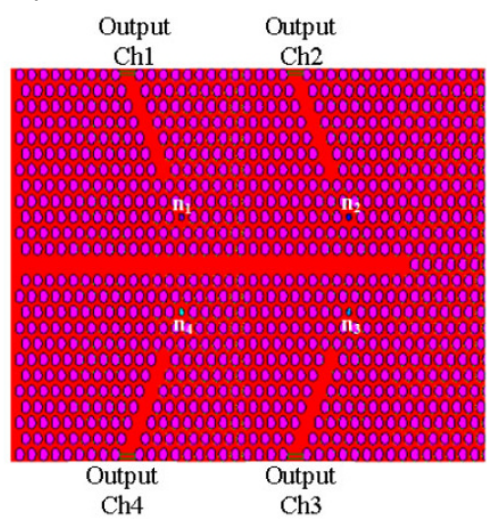

Fig. 1 Two-dimensional four-channel photonic crystal biosensor structure. 
The second: four nano-cavities which are filled with different refractive indices of $n_{1}, n_{2}, n_{3}$, and $n_{4}$, respectively, are used for trapping the special wavelength in proportion to the injected bio-molecule, as shown in Fig. 1.

The third: four waveguides which are called drop waveguides [20] are formed in order to detect trapped resonant modes in any nano-cavity. The biosensor is designed by the hexagonal array of air holes with the lattice constant of $a, 400 \mathrm{~nm}$ and the radius of $140 \mathrm{~nm}$ with the refractive index $n$ of 1 in the silicon slab with $n$ of 3.45 . The nano-cavities sizes are optimized for achieving the high quality factor, high transmission efficiency, and reasonable sensitivity. The two-dimensional finite-difference time-domain (2-D FDTD) method and plane-wave expansion (PWE) approach are used for analyzing this sensor. The photonic band gap range for transverse electric (TE) modes for the periodic structure obtained by the PWE method is 0.22054 to 0.32550 as shown in Fig. 2.

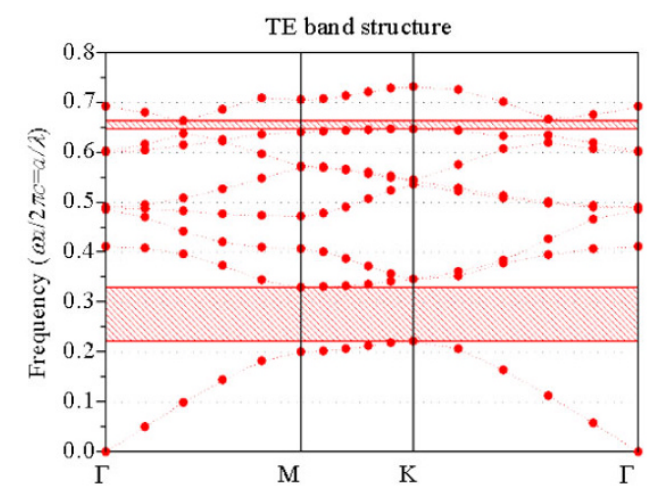

Fig. 2 Band structure of the two-dimensional four-channel photonic crystal biosensor.

This is a forbidden frequency range which cannot propagate through the structure. But after introducing defects like refractive index variation or hole radius modifying which break the periodicity of the structure, an allowable frequency range is created that can propagate through the structure within the PBG. This property is utilized for sensing applications in the study of the resonance wavelength value and calculation of the sensitivity and quality factor, which are shown in the next simulations.

For having the high performance, the perfectly matched layer (PML) with the 500-nm width is considered. The grid size in the $x$ - and $z$-directions are set to $\Delta x=\Delta z=20 \mathrm{~nm}$. For achieving the stability condition, the time step is determined by [9]

$$
\Delta t=\frac{1}{c \sqrt{\left(\frac{1}{\Delta x^{2}}\right)+\left(\frac{1}{\Delta z^{2}}\right)}}
$$

where $c$ is the speed of light in the free space.

\section{Evaluating the biosensing characteristics}

In the bio-sensing application, the device is not utilized for measuring bulk refractive index variations of the around ambient, whereas it is utilized for measuring local refractive index variations. Hence, when we regard that the refractive index (RI) of the nano-cavities has been changed, it means that the target molecules flow in an aqueous medium. The presence of target molecules varies the ERI of the sensing hole. The change in the ERI is utilized to calculate the presence of target molecules. This sensing mechanism is based on homogenous sensing. So the output spectrum is changed. As a result, the spectral shift is afforded due to ERI variations of the defect.

In this section, we suppose $n_{1}=1.25, n_{2}=1.5$, $n_{3}=1.75$, and $n_{4}=2$. With the variation of the nano-cavities size between $78 \mathrm{~nm}$ and $88 \mathrm{~nm}$, the resonant wavelength changes with respect to the RI variation as shown in Fig. 3(a). The output transmission efficiency and quality factor of the four-channel biosensor are respectively shown in Figs. 3(b) and 3(c).

As depicted in Fig. 3(a), with increasing the defect size, the slope of the diagram that shows the sensitivity increases so that the lowest dashed curve which is the characteristic of $R_{c}=88 \mathrm{~nm}$ has the high sensitivity in comparison with others, and the highest dashed curve for $R_{c}=78 \mathrm{~nm}$ has the lower sensitivity. Also, the length of waveguides, size of 
the crystal, numbers of periods between the nano-cavity and the waveguides are selected so that the high quality factor and transmission efficiency have been achieved.

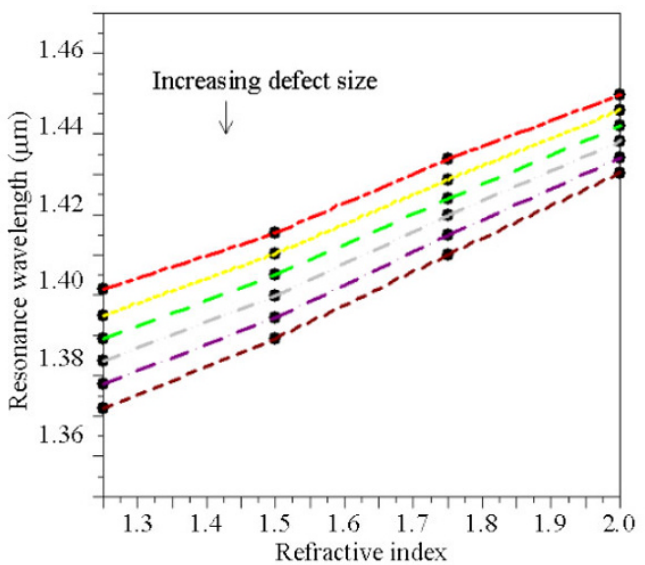

(a)

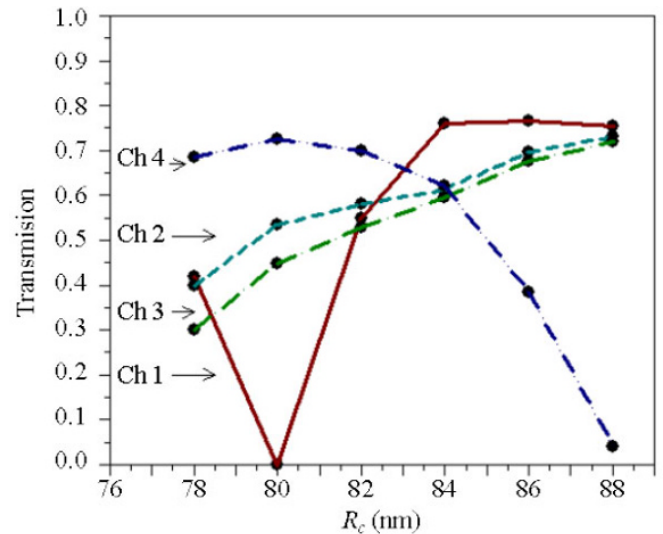

(b)

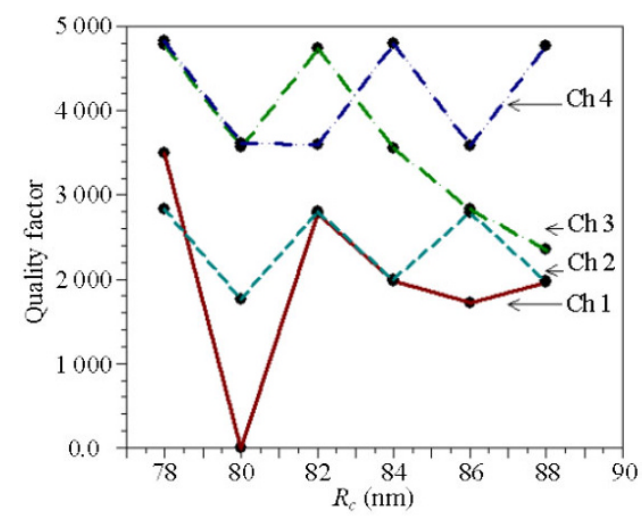

(c)

Fig. 3 Illustration of (a) the resonant wavelength variation with respect to the refractive index, (b) the transmission efficiency, and (c) the quality factor of channels for the nanocavity size of $R_{c}$ in unit of $\mathrm{nm}$.
With regard to Fig. 3, when the defect sizes are $80 \mathrm{~nm}$ and $88 \mathrm{~nm}$, their transmission efficiency in one of channels is very small, and also for $R_{c}=78 \mathrm{~nm}$, although the structure has the high quality factor, the transmission efficiency and sensitivity are low, so these radii could not be selected. Among $R_{c}=86 \mathrm{~nm}$, $84 \mathrm{~nm}$, and $82 \mathrm{~nm}$, we select $R_{c}=84 \mathrm{~nm}$ as the optimum radius because for $R_{c}=86 \mathrm{~nm}$, the sensitivity is larger than $R_{c}=84 \mathrm{~nm}$ and $82 \mathrm{~nm}$ but the $Q$-value is low, and for $R_{c}=82 \mathrm{~nm}$, the $Q$-value is larger than $R_{c}=86 \mathrm{~nm}$ and $84 \mathrm{~nm}$ but the sensitivity is low.

In this research, with regard to simulation results, $R_{c}=84 \mathrm{~nm}$ was selected as the optimum radius of nano-cavities, because for this radius the favorite outputs were achieved with the high quality factor, good transmission efficiency, and reasonable sensitivity which could make detection of bio-chemicals easy and possible. The resonant wavelength of nano-cavities when no analyte was filled within the holes was obtained as $1.3724 \mu \mathrm{m}$.

The transmission spectra of four-channel outputs are depicted in Fig. 4. According to Fig. 4, with increasing the refractive index of nano-cavities, the transmission spectrum shifts to longer wavelengths.

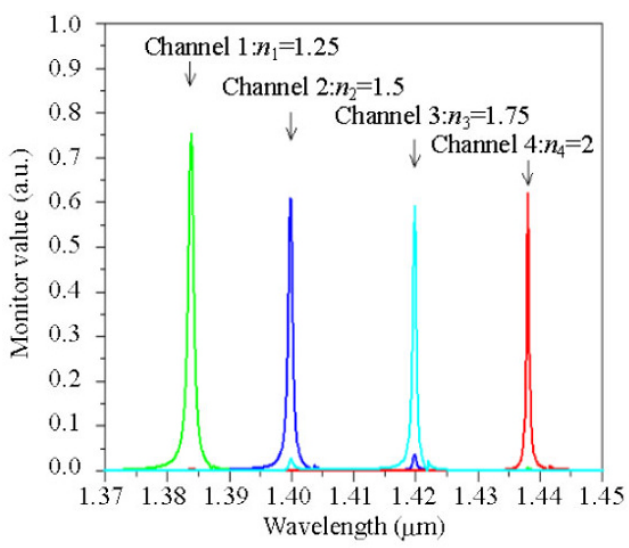

Fig. 4 Transmission spectra of the designed four-channel biosensor with the defect size $R_{c}=84 \mathrm{~nm}$.

The results for the novel designed multi-channel biosensor are listed in Table 1. According to this table, $\lambda_{0}$ characterizes the central wavelength of each channel, and $\Delta \lambda_{\text {FWнм }}$ is the full width at the 
half-maximum (FWHM) of the output which indicates the narrow band biosensor behavior. According to $\lambda_{0}$ and $\Delta \lambda_{\text {FWHM }}$, the values of quality factors for each channel are calculated as $\left(\lambda_{0} / \Delta \lambda_{\text {FWHM }}\right)$, and the transmission efficiency is defined as the division of the output magnetic field amplitude to the input magnetic field amplitude. And also $\Delta \lambda$ is the resonant wavelength shift from the reference state (no analyte is filled within the holes) that shows the mass sensitivity.

Table 1 Simulation results of the biosensor with the defect size $R_{c}=84 \mathrm{~nm}$.

\begin{tabular}{ccccc}
\hline Channel & Channel 1 & Channel 2 & Channel 3 & Channel 4 \\
\hline Refractive index & $n_{1}=1.25$ & $n_{2}=1.5$ & $n_{3}=1.75$ & $n_{4}=2$ \\
$\lambda_{0}(\mu \mathrm{m})$ & 1.3837 & 1.3999 & 1.4198 & 1.4381 \\
$\Delta \lambda_{\text {FWHM }}(\mathrm{nm})$ & 0.7 & 0.7 & 0.4 & 0.3 \\
Quality factor & 1976.71 & 1999.86 & 3549.5 & 4793.6 \\
$\Delta \lambda(\mathrm{nm})$ & 11.3 & 27.5 & 47.4 & 65.7 \\
Efficiency & $76.59 \%$ & $61.27 \%$ & $59.53 \%$ & $62.13 \%$ \\
\hline
\end{tabular}

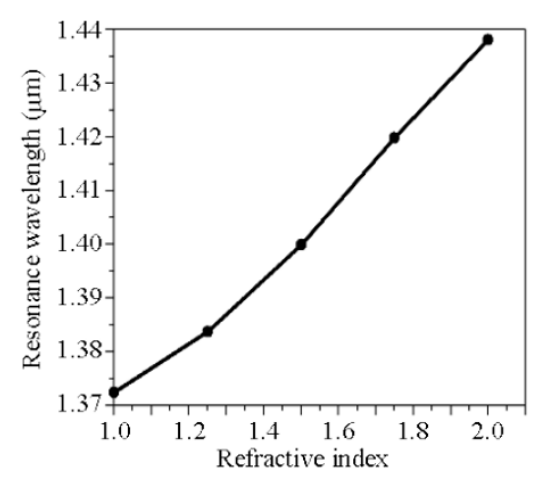

Fig. 5 Resonant wavelength with respect to the refractive index for each channel.

Figure 5 indicates the resonant wavelength with respect to the RI for the defect size of $R_{c}=84 \mathrm{~nm}$. It is shown that with increment of the RI, the resonant wavelength shifts to longer wavelengths. Therefore, we can calculate the sensitivity that is defined as the wavelength shift per refractive index unit $(\Delta \lambda / \Delta n)$ equal to $65.7 \mathrm{~nm} / \mathrm{RIU}$. In previous researches, the structure of the multi-channel biosensor was based on micro-cavities and waveguides, our present structure is based on nano-cavities and waveguides in a wide measurement range. The small and compact structures are necessary because generally the amount of bio-materials in bio-sensing applications is low. Also miniaturization sensing elements allow implementing compact and optical integrated chips.

\section{Conclusions}

In conclusion, a novel compact four-channel biosensor has been designed. Single purpose operation of the sensor and amount of detected target molecules were limitations which motivated us to design a multi-channel biosensor. The channels of this sensor can measure four different refractive indices in a special time. The structure has been assumed to be embeded in a micro-fluidic channel for inducting the analyte. The biosensing mechanism is based on the effective refractive index change of the sensing hole. The bio-molecules have been filled in a special hole within the nano-cavities and caused the ERI variation of the hole and resonant wavelength shift in the output terminal. The structure has been optimized in characteristics of the high quality factor, high transmission efficiency, and reasonable sensitivity with the approprate mass sensitivity. By filling an analyte into the nanocavity, the resonant wavelength shifted, and this process was utilized for determining the properties of the analyte. The results revealed that by increasing the radius of the nanocavity, the sensitivity was improved, and by decreasing this radius, the quality factor was increased. Therefore, we have choosen an optimum state.

Open Access This article is distributed under the terms of the Creative Commons Attribution License which permits any use, distribution, and reproduction in any medium, provided the original author(s) and source are credited. 


\section{References}

[1] J. D. Joannopoulos, S. G. Johnson, J. N. Winn, and R. D. Meade, Photonic crystals molding the flow of light. Princeton: Princeton University Press, 2007.

[2] J. M. Lourtioz, "Photonic crystals and metamaterials," Comptes Rendus Physique, vol. 9, no. 1, pp. 4-15, 2008.

[3] J. M. Lourtioz, H. Benisty, V. Berger, J. M. Gerard, D. Maystre, and A. Tchelnokov, Photonic crystals towards nanoscale photonic devices. Berlin: SpringerVerlag Berlin Heidelberg, 2008.

[4] S. Olyaee and F. Taghipour, "Ultra-flattened dispersion hexagonal photonic crystal fiber with low confinement loss and large effective area," IET Optoelectronics, vol. 6, no. 2, pp. 82-87, 2012.

[5] S. Olyaee and A. A. Dehghani, "High resolution and wide dynamic range pressure sensor based on two-dimensional photonic crystal," Photonic Sensors, vol. 2, no. 1, pp. 92-96, 2012.

[6] A. Saynatjoki, M. Mulot, K. Vynck, D. Cassagne, J. Ahopelto, and H. Lipsanen, "Properties, applications and fabrication of photonic crystals with ring-shaped holes in silicon-on-insulator," Photonics and Nanostructures, vol. 6, no. 1, pp. 42-46, 2008.

[7] J. Sun and C. C. Chan, "Photonic bandgap fiber for refractive index measurement," Sensors and Actuators B: Chemical, vol. 128, no. 1, pp. 46-50, 2007.

[8] R. V. Nair and R. Vijaya, "Photonic crystal sensors: an overview," Progress in Quantum Electronics, vol. 34, no. 3, pp. 89-134, 2010.

[9] A. Rostami, H. A. Banaei, F. Nazari, and A. Bahrami, "An ultra compact photonic crystal wavelength division demultiplexer using resonance cavities in a modified Y-branch structure," Optik, vol. 122, no. 16, pp. 1481-1485, 2011.

[10] E. Hallynck and P. Biensman, "Photonic crystal biosensor based on angular spectrum analysis," Optics Express, vol. 18, no. 17, pp. 18164-18170, 2010.

[11] W. Zhang, N. Ganesh, I. D. Block, and B. T. Cunningham, "High sensitivity photonic crystal biosensor incorporating nanorod structures for enhanced surface area," Sensors and Actuators B: Chemical, vol. 131, no. 1, pp. 279-284, 2008.

[12] J. Derbali, F. A. Malek, S. S. A. Obayya, H. Bouchriha, and R. Letizia, "Design of a compact photonic crystal sensor," Optical and Quantum Electronics, vol. 42, no. 8, pp. 463-472, 2010.

[13] X. Fan, I. M. White, S. I. Shopova, H. Zhu, J. D. Suter, and Y. Sun, "Sensitive optical biosensors for unlabeled targets: a review," Analytical Chimica Acta, vol. 620, no. 1-2, pp. 8-26, 2008 .

[14]L. L. Chan, S. L. Gosangari , K. L. Watkin, and B. T. Cunningham, "A label-free photonic crystal biosensor imaging method for detection of cancer cell cytotoxicity and proliferation," Apoptosis, vol. 12, no. 6, pp. 1061-1068, 2007.

[15] F. Hsiao and C. Lee, "Computational study of photonic crystals nano-ring resonator for biochemical sensing," IEEE Sensors Journal, vol. 10, no. 7, pp.1185-1191, 2010.

[16] K. Manoharan, "Design and analysis of high-Q, amorphous microring resonator sensors for gaseous and biological species detection," M.S. thesis, Russ College of Engineering and Technology of Ohio University, Mar., 2009.

[17] S. H. Kwon, T. Sünner, M. Kamp, and A. Forchel, "Optimization of photonic crystal cavity for chemical sensing," Optics Express, vol. 16, no. 16, pp. 11709-11717, 2008.

[18] X. Wang, Z. Xu, N. Lu, J. Zhu, and G. Jin, "Ultracompact refractive index sensor based on microcavity in the sandwiched photonic crystal waveguide structure," Optics Communications, vol. 281, no. 6, pp. 1725-1731, 2008.

[19] D. Q. Yang H. P. Tian, and Y. F. Ji, "Nanoscale photonic crystal sensor arrays on monolithic substrates using side-coupled resonant cavity arrays," Optics Express, vol. 19, no. 21, pp. 20023-20034, 2011.

[20] F. Hsiao and C. Lee, "Novel biosensor based on photonic crystal nano-ring resonator," Procedia Chemistry, vol. 1, no. 1, pp. 417-420, 2009. 